\title{
Effect of Self-Microemulsifying Lipid Formulations on the Dissolution and Compaction Profiles of Tablets Containing Theophylline; A BCS Class I Compound
}

\author{
Naser M.Y. Hasan ${ }^{1 *}$, Mohammad A. Khaleel ${ }^{2}$, Abdullah S. Altwairqi ${ }^{2}$, Abdulmajeed G. Alqurashi ${ }^{2}$, Abduallah H. Altwairqi ${ }^{2}$ \\ ${ }^{1}$ School of Pharmacy, Applied Science Private University, Jordan. \\ ${ }^{2}$ School of Pharmacy, Taif University, Saudi Arabia.
}

\begin{tabular}{|c|c|}
\hline ARTICLE INFO & ABSTRACT \\
\hline Article history: & \multirow{5}{*}{$\begin{array}{l}\text { There is growing interest amongst formulation scientists to use self-emulsifying lipid technology as an approach } \\
\text { to improve dissolution and hence absorption of poorly-water soluble drugs. Nonetheless, lipid-based formulations } \\
\text { require that oil content is encapsulated in soft gelatin capsules which might exhibit some disadvantages. To overcome } \\
\text { these limitations, solid self-micro-emulsifying drug delivery system (S-SMEDDS) is introduced as an alternative } \\
\text { approach. The preferred dosage form for BCS class I compounds is tablets yet, some drugs which belong to this } \\
\text { category might suffer from enzymatic degradation and gut wall efflux. The effect of converting liquid SMEDDS into } \\
\text { compressed tablets containing a BCS class } 1 \text { compound is investigated. A SMEDDS oil formulation representing type } \\
\text { III A lipid class was converted into S-SMEDDS using solid carrier adsorption method and compressed into tablets } \\
\text { containing theophylline. The effect of oil loading factor on compressibility, disintegration and dissolution kinetics of } \\
\text { theophylline from various tablet formulation was investigated. Increasing oil content in the compressed tablets ensued } \\
\text { in a progressive decrease in the hardness of tablets. Analysis of the dissolution kinetic data for tested theophylline } \\
\text { preparations shows that it follows first-order or Higuchi kinetics. Fast dissolving tablet formulations were obtained at } \\
\text { including an optimum oil concentration of } 5 \% \mathrm{w} / \mathrm{w} \text {. }\end{array}$} \\
\hline Received on: 29/10/2017 & \\
\hline Accepted on: 30/12/2017 & \\
\hline Available online: $29 / 06 / 2018$ & \\
\hline $\begin{array}{l}\text { Key words: } \\
\text { SMEDDS, Lipid } \\
\text { formulations, Solid } \\
\text { SMEDDS, Theophylline, } \\
\text { Dissolution. }\end{array}$ & \\
\hline
\end{tabular}

\section{INTRODUCTION}

There is growing interest amongst formulation scientists in the field of self-emulsifying lipid technology as an approach to improve dissolution and hence absorption of poorly-water soluble drugs. This has attracted pharmaceutical industry as almost $60 \%$ of newly discovered APIs are classified as class II drugs according to Biopharmaceutics Classification System (Khairnar et al., 2016). As a result of this mature technology, the market has witnessed the introduction of several products including; Neoral ${ }^{\circledR}$ (cyclosporine A/I; immune suppressant by Novartis), Norvir ${ }^{\circledR}$ (ritonovir; HIV antiviral by Abbott Laboratories), Agenerase ${ }^{\circledR}$ (amprenavir; HIV antiviral by Glaxo Smithlkine), Lipirex ${ }^{\circledR}$ (fenofibrate; antihyperlipoproteineic by Genus), Targretin ${ }^{\circledR}$ (bexarotene;

"Corresponding Author

Naser M.Y. Hasan, School of Pharmacy, Applied Science Private

University,Jordan.E-mail:n_hassan@asu.edu.jo antineoplastic by Ligand), Rocaltrol ${ }^{\circledR}$ (calcitriol, calcium regulator by Roche) and Gengraf ${ }^{\circledR}$ (cyclosporine A/III; immune suppressant by Abbott Laboratories) (Shraddha et al., 2016).

Biopharmaceutics Classification System (BCS) is considered a tool to characterize drug permeability and solubility/ dissolution (Brouwers et al., 2010; Butler and Dressman, 2010). According to $\mathrm{BCS}$, drugs are characterized into four categories: Class I-high permeability, high solubility; Class II-high permeability, low solubility; Class III—low permeability, high solubility; Class IV - low permeability, low solubility. According to the Food and Drug Administration (FDA) guidance, a drug substance is considered as high soluble when the highest dose strength is soluble in $\leq 250 \mathrm{~mL}$ of aqueous media over the $\mathrm{pH}$ range of 1.0-7.5 (U.S. FDA, 2000). A drug substance is considered to be "highly permeable" when $\geq 90 \%$ of an administered dose is absorbed in humans based on a mass balance determination or in comparison to an intravenous reference dose (U.S. FDA, 
2015). Self-emulsifying lipid technology (SELT) includes selfemulsifying drug delivery systems (SEDDS) and self-microemulsifying drug delivery system (SMEDDS) or sometimes is referred to as self-nano-emulsifying drug delivery systems (SNEDDS). SEDDS are isotropic mixtures of oils and nonionic surfactants which produce $(\mathrm{o} / \mathrm{w})$ dispersions of droplets $<5 \mu \mathrm{m}$ upon gentle agitation in water (Shah et al., 1994). As for SMEDDS/SNEDDS, the vehicle is, nonetheless, more hydrophilic composed of oils or modified oils, surfactant and co-surfactant mixtures which emulsifies spontaneously when mixed with water under gentle agitation forming a o/w microemulsion of droplets with diameters between 5 and $140 \mathrm{~nm}$ (Farah et al., 1993). Both SEDDS and SMEDDS/SNEDDS can enhance bioavailability of lipophilic drugs as they provide a reservoir of drug dissolved in the lipid matrix, which spontaneously emulsifies on contact with gastrointestinal fluids producing oil-in-water dispersions of small particle size with the large surface area available for drug diffusion (Hasan, 2004). Furthermore, the varying constituents of these lipid vehicles can affect membrane permeability and hence these excipients can act as intestinal absorption enhancers by weakening the tight junction of paracellular membrane (Buyukozturk and Benneyan, 2010), interact with intestinal-based drug transporter (P-gp efflux) and metabolic processes (CYP3A4) (Bansal et al., 2009; Nornoo et al., 2009) and moreover, can influence absorption pathways by initiating lymphatic transport (Jannin et al., 2008; Nankervis et al., 1995; Porter et al., 2004; Sha et al., 2012). Therefore, it is anticipated that BCS class II, III or IV compounds can benefit from the formulation design of SELT. Nonetheless, lipid-based formulations require that oil content be encapsulated in soft gelatin capsules or more recently into two-piece hard gelatin capsules such as Licaps ${ }^{\circledR}$ developed by Capsugel. This might, however, raise some physical and chemical stability concerns including; possible interaction between the filling and the capsule shell, precipitation of either active ingredient and/or oil constituents as influenced by storage temperature and high production cost (Sharma et al., 2013; Hasan, 2015a, Hasan et al., 2015b). Recently, nonetheless, the concept of Solid SEDDS or SMEDDS/SNEDDS has emerged as an alternative approach to overcome these limitations. Yet, there is still some doubt concerning reliability for the commercial production of solid lipid systems, as the whole idea of SELT is to administer the drug in an already dissolved form within lipid matrix. This allows the drug to be absorbed without disintegration and dissolution steps, which are quintessential for the absorption of solid self-emulsifying systems and hence, this will compromise bioavailability of such systems. Furthermore, there are many discrepancies with regards to the pharmacokinetic profiles of solid SNEDDS (S-SNEDDS) versus conventional liquid SNEDDS (L-SNEDDS) (Chatterjee et al., 2016). In one study using darunavir, a poorly soluble drug has shown a higher extent of absorption and bioavailability from S-SNEDDS compared to L-SNEDDS (Inugala et al., 2015). Another study using olmesartan medoxomil as a drug both S-SNEDDS and L-SNEDDS have shown same release kinetics. On the other hand, cinnarizine showed lesser oral bioavailability in beagle dogs from S-SMEDDS than L-SNEDDS filled into capsules (Christiansen et al., 2014). Therefore, it is thought that solid self-emulsifying systems from formulation aspects and stability have an advantage. Yet, in terms of pharmacokinetics benefits, it cannot be concluded that solid SEDDS is much better than liquid SEDDS filled capsule (Chatterjee et al., 2016).

Solid SEDDS or SMEDDS are developed by converting the liquid or semisolid formulation into powders by using various techniques such as; spray drying or freeze drying (Tang et al., 2013; Eng and $\mathrm{Xu}, 2008)$, adsorption to solid porous carriers (Kang et al., 2011; Laddha et al., 2014), solid dispersions (Hasan et al., 2015b; Okimoto et al., 1997), extrusion spheronization (Abdulla et al., 2008; Setthacheewakul et al., 2010) and melt granulation (Chambin et al., 2004). Solid SEDDS can be, therefore, formulated into free-flowing powders, granules, pellets, tablets, solid dispersions, microspheres, and nanoparticles. There are many drugs in the literature which are formulated as orally delivered solid SEDDS/SNEDD using various methods to carry out comparative pharmacokinetic parameters analysis. This includes; Lutein (Shanmugam et al., 2011), docetaxel (Quan et al., 2013), flurbiprofen (Kim et al., 2012) and clopidogrel (Kim et al., 2014); using spray drying technique, isradipine (Ramasahayam et al., 2015) and cyclosporine-A (Zaho et al., 2011; Sander and Holm, 2008) using solid carrier adsorption method.

As for BCS class I compounds, such as amiloride, theophylline, captopril and diazepam, the preferred dosage form is tablets. However, some drugs which belong to this category might suffer from enzymatic degradation and gut wall efflux and hence this might compromise bioavailability (Shraddha et al., 2016). Many surfactants like tween 80, spans, cremophors (EL and RH40) and pluronics which are used in the formulation design of SEDDD or SMEDDS may reduce efflux of the drug in the GIT due to their inhibitory effect on to p-glycoprotein transporter which ensues in improving the bioavailability of the drugs (Bansal et al., 2009; Nornoo et al., 2009). Therefore, the drugs which have the propensity to be effluxed from the GIT can be formulated as lipidbased delivery systems for the improvement of bioavailability (Khairnar et al., 2016; Gohel, 2011).

There are not many reports in the literature which study the effect of SEDDS or SMEDDS on the physico-mechanical behavior and release patterns of powder compacts containing $\mathrm{BCS}$ class I drugs. Theophylline was selected here and used in this study as a model drug representing BCS class I compound. In this investigation, a SMEDDS oil formulation representing type III A class system was converted into solid SMEDDS by adsorbing unto porous solid carrier at various oil loading factors and blended with the drug and other pharmaceutical excipients and then compressed into tablets. Effect of the amount of lipidic formulation included in the powder blend and compression loading force on the mechanical strength, disintegration and dissolution profiles of the compressed tablets containing theophylline were carried out on an attempt to expand applications of SMEDDS in the field of solid dosage forms.

\section{MATERIALS AND METHODS}

\section{Materials}

Glycerox 767HC (PEG 6 caprylic/capric glycerides) and croduret 40ss (PEG 40 hydrogenated castor oil) were all supplied by Croda as gift samples. Magnesium trisilicate hydrate from Sigma and theophylline anhydrate was supplied by Himedia, India. Microcrystalline cellulose $50 \mu \mathrm{m}$ grade was purchased from Organics. 


\section{Methods}

\section{Preparing oil SMEDDS mixtures}

Croduret 40ss was first thawed at $70^{\circ} \mathrm{C}$. Mixtures of glycerox $767 \mathrm{HC}$ and croduret 40 ss at ratios of 8:2 were accurately weighed into $20 \mathrm{ml}$ capped glass vials and then hermetically sealed by cling film followed by vortexing. Glass vials were held at $50^{\circ} \mathrm{C}$ in a thermostated water bath held for 2 minutes before lipid mixtures were thoroughly vortexed. Lipid formulations were then left to equilibrate overnight at room temperature and kept as a stock to be used whenever is needed.

\section{Converting L-SMEDDS into S-SMEDDS}

The prepared oil SMEDSS which is composed of glycerox $767 \mathrm{HC}$ and croduret 40ss at ratios of 8:2 was mixed with magnesium trisilicate hydrate (MTSH) at oil loading factors of $\{1: 9\},\{2: 8\}$ or $\{3: 7\}$, then admixed with microcrystalline cellulose and magnesium stearate to obtain formulations F0, F1 or F2, respectively; see Table 1 for formulations composition. Theophylline was added to the three types of formulations at a ratio of $20 \% \mathrm{w} / \mathrm{w}$.

Table 1: Composition of various types of formulations used in this investigation.

\begin{tabular}{ccccc}
\hline & \multicolumn{4}{c}{ Components } \\
\cline { 2 - 5 } Formula & $\begin{array}{c}\text { SMEDDS } \\
\text { Oil \% }\end{array}$ & $\begin{array}{c}\text { Mg silicate } \\
\text { hydrate \% }\end{array}$ & MCC \% & Mg stearate \% \\
\hline F0 & 0 & 50 & 49 & 1 \\
F1 & 5 & 45 & 49 & 1 \\
F2 & 10 & 40 & 49 & 1 \\
\hline
\end{tabular}

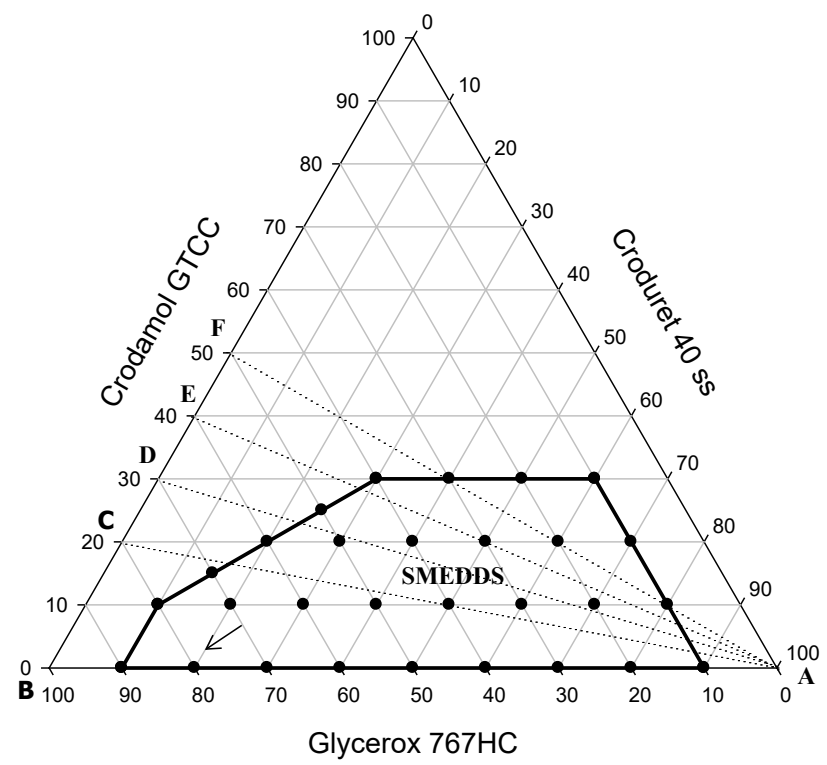

Fig. 1: Emulsification profile of a lipid system composed of crodamol GTCC (oil), glycerox 767HC (co-surfactant) and croduret 40ss (non-ionic surfactant) (Hasan et al., 2015c).

\section{Preparing tablet SMEDDS}

Various powder mixtures representing the various types of formulations; F0, F1, F2 with and without drug were compressed into tablets on Erweka single punch tableting machine
(EP-1 vers-2), using Adamus $(01 / 1216 \times 8 \mathrm{~mm})$ punch. Average tablet weight was $0.65 \pm 0.025 \mathrm{~g}$. Theophylline content in each tablet was equivalent to approximately $125 \mathrm{mg}$.

Preparing solid SMEDDS compacts (tablet SMEDDS) at varying loading compression forces

An amount of $1.2 \mathrm{~g}$ of various powder mixtures representing the various types of formulations; F0, F1, F2 without drug was added to static loading cell attached to Instron universal testing system and compressed at loading forces of 3, 5, $10 \mathrm{KN}$. The retrieved solid SMEDSS compacts were tested for hardness.

\section{Measuring tablet hardness}

Tablet hardness was determined on tablets compressed at $1.5 \mathrm{~m}$ ton for 3 seconds on Erweka $\mathrm{GmbH}$ tablet hardness machine (TBH 225, Germany).

\section{Calibration of theophylline}

A calibration curve was constructed according to the method described in USP 39 by making series of dilutions in water to obtain theophylline concentrations ranging from $1 \mu \mathrm{g}$ to $30 \mu \mathrm{g}$. The absorbance of the various solutions was measured by UV spectrophotometry at $\lambda \max 272 \mathrm{~nm}$ using SP-3000 nano optima.

\section{Disintegration of tablets}

Total tablets of 6 from each formulation were inserted into the baskets of DST 3 automatic disintegration testing apparatus (Logan Instruments Corp.) A beaker containing $1000 \mathrm{ml}$ of water previously heated to $37 \pm 2{ }^{\circ} \mathrm{C}$ was put in place according to the method described in the USP 39 . The motor rotation speed was set at $30 \mathrm{rpm}$. 4. Insert the tablet, turn on the motor and begin to measure the disintegration time 5 . Observe visually the course of the test. Disintegration time for each tablet when there is no residue of the tablet left in the basket was recorded and the average was calculated $(n=6)$.

\section{Dissolution of theophylline tablets}

Dissolution of theophylline tablets was carried according to the methods described in the USP 39 monograph. Erweka Dissolution apparatus, USP dissolution paddle, containing $900 \mathrm{ml}$ distilled water as a dissolution media was used. The temperature of dissolution medium was controlled at $37 \pm 0.5^{\circ}$, and the stirring speed was maintained at $50 \mathrm{rpm}$. Two tablets from each batch were tested for a period of one hour. One $\mathrm{ml}$ aliquot samples were withdrawn at predetermined time intervals and then filtered using $0.45 \mu \mathrm{m}$ pore size syringe filters. Collected samples were diluted and then assayed by UV spectrophotometry at $\lambda \max 272 \mathrm{~nm}$ to measure the cumulative concentration of dissolved theophylline.

\section{RESULTS AND DISCUSSION}

\section{Effect of applied force on the hardness profiles of solid SMEDDS (S-SMEDDS)}

Self-micro-emulsifying lipid systems were classified by Pouton (2006) into type I, II IIIA, IIIB and IV according to the hydrophilicity of oil mixture, oil droplet size after aqueous dispersion and digestion by bile salts. As you move from Class I lipid system to class IV, hydrophilic content (co-surfactants/hydrophilic 
co-solvents) of the lipid composition increases on the account of hydrophobic lipidic content (source triglycerides). Therefore, type IV systems represent the most hydrophilic formulations as they do not contain natural lipids. An archetypal example of a Type IV formulation is the current capsule formulation of the HIV protease

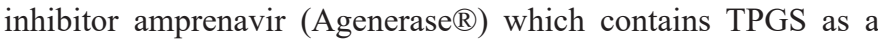
surfactant and PEG 400 and propylene glycol as co-solvents.

(a)

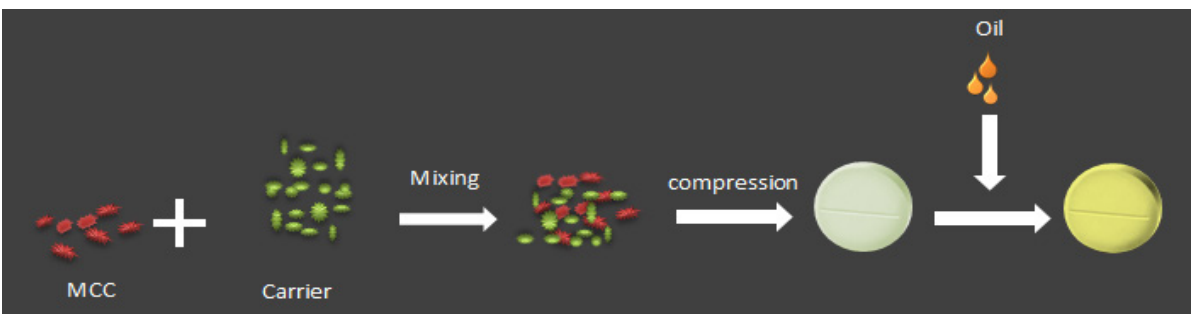

(b)
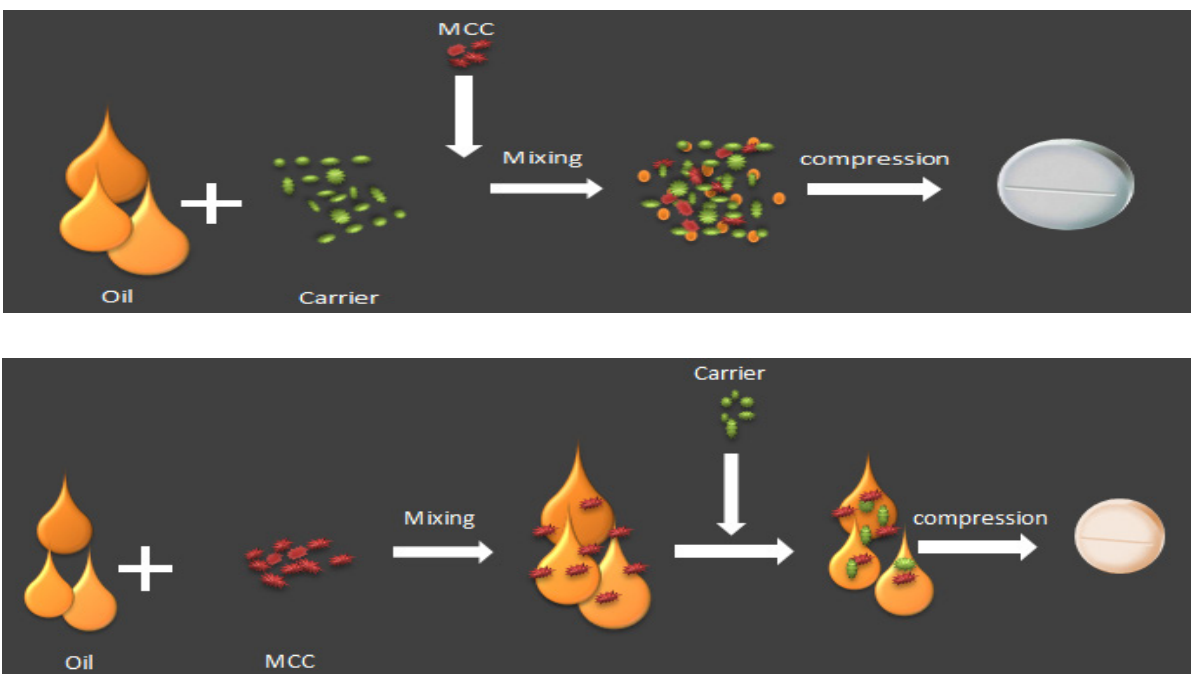

Fig. 2: Various approaches to convert liquid SMEDD (L-SMEDDS) into tablet SMEDDS using solid carrier adsorption technique.

In this investigation, based on previous research by our team (Hasan et al., 2015c), a self-micro-emulsifying oil formulation representing type III A lipid class composed of Glycerox $767 \mathrm{HC} /$ Croduret 40 ss at ratios of (80/20) was selected as, shown in Figure 1 . This system has the advantage of high solubilization capacity of drugs, forming clear microemulsion on aqueous dilution and also the ability to retain its solvent capacity and hence circumvents drug precipitation after dispersion of the formulation. This system was converted into solid self-micro-emulsifying drug delivery system (S-SMEDDS) using solid carrier adsorption technique which was then compressed into tablets. In the literature, there are three approaches to convert liquid SMEDD (L-SMEDDS) into tablet SMEDDS, as the diagram depicted in Figure 2 shows. (A) Microcrystalline cellulose (MCC) is mixed with the porous solid carrier, compressed into tablets and then L-SMEDDS containing the dissolved drug is adsorbed onto the surface of the tablet. (B) L-SMEDDS is added to the solid carried at the required ratio which is identified as the oil loading factor; MCC is then added to the powder mix and compressed into tablets. (C) L-SMEDDS is mixed with MCC and the adsorbent solid carried is then added to the powder mix and compressed into tablets. In this investigation, second approach was used to convert L-SMEDDS into compressed tablets. Microcrystalline cellulose (MCC) due to its outstanding dry binding properties is considered a key tableting diluents, enabling the manufacture of tablets by direct compression (DC) (Gregory et al., 2014). Hydrogen bonds on adjacent cellulose molecules solely account for strength and cohesiveness. MCC particles are plastically deformed under compaction forces to yield an extremely large number of clean surfaces brought in contact during this deformation forming a strong compact even under low compression forces (Schwartz and Lachman, 1990). Owing to its relatively high bulk and tapped density and furthermore, due to water of hydration present in its molecules, Magnesium trisilicate hydrate, as an adsorbent solid carrier, has shown relatively high compressibility with MCC than Magnesium Aluminum silicate (Hasan et al., 2016). Therefore, Magnesium trisilicate hydrate was used in this study as a solid adsorbent carrier.

Contour plot depicted in Figure 3 shows the effect of applied compression force on the mechanical strength of compressed tablets containing a fixed amount of oil: adsorbent carrier (identified as oil loading factor), at a ratio of $\{1: 9\}$ with varying amounts of MCC. As the applied compression force increases, the hardness of compressed tablets increases accordingly. At fixed applied force, relatively high values of the mechanical strength of the compressed tablets are observed when the powder mix relatively contains larger amounts of the MCC. Similar hardness profiles are observed in the case of using higher amounts of oil in the compressed tablets i.e. oil: adsorbent carrier at a ratio of $\{2: 8\}$, as illustrated in the contour plot presented in Figure 4. However, increasing the amount of SMEDDS oil which is added to the powder compact from $5 \%$ to $10 \% \mathrm{w} / \mathrm{w}$ has resulted in a drop in the mechanical strength of tablets. The maximum 
value of tablet hardness when using oil: adsorbent carrier at a ratio of $\{1: 9\}$ added to the powder mix is approximately $200 \mathrm{KN}$, in comparison to $140 \mathrm{KN}$ obtained when using oil loading factor at a ratio of $\{2: 8\}$.

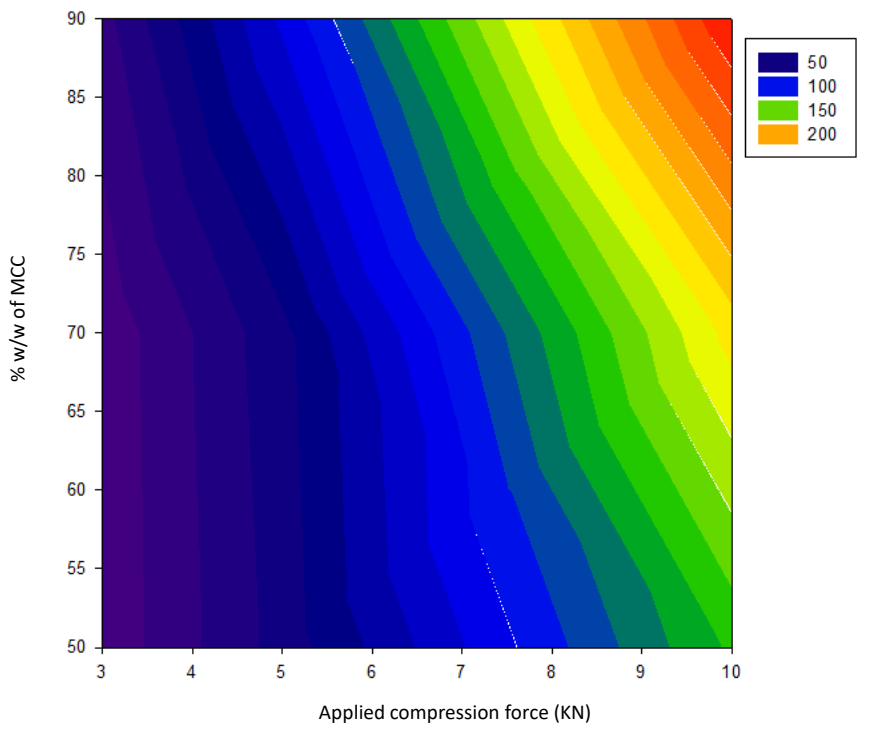

Fig. 3: Contour plot of the effect of applied compression force on the mechanical strength of compressed tablets using oil loading factor at a ratio of $\{1: 9\}$ with varying amounts of MCC.

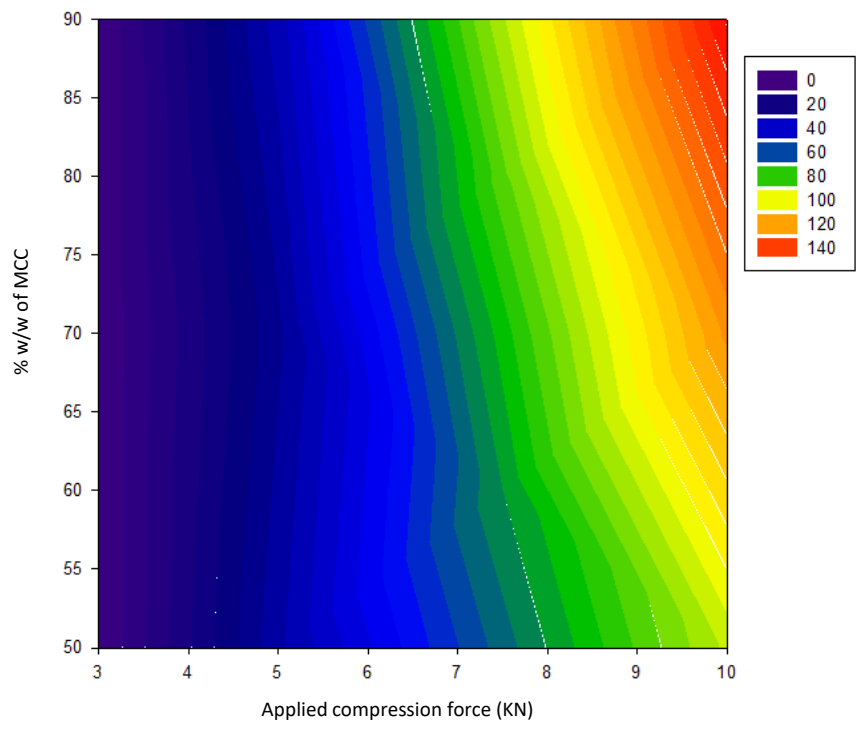

Fig. 4: Contour plot of the effect of applied compression force on the mechanical strength of compressed tablets using oil loading factor at a ratio of $\{2: 8\}$ with varying amounts of MCC.

\section{Effect of amount of oil on the hardness profiles of S-SMEDDS}

Oil loading factor can be identified here as the amount of oil adsorbed unto carrier i.e. ratio of added oil to the adsorbent carrier. In a recent study by Hasan et al. (2016) has shown that adding as little as $4 \% \mathrm{w} / \mathrm{w}$ of lipidic oil to the powder compact could affect the physical strength of tablets. This is evident from Figure 5 and the contour plot depicted in Figure 6 which show a gradual and sharp drop in the hardness of compressed tablet as more amounts of lipidic SMEDDS are added to the powder mix. It is thought that the added oil would soften powder compacts and furthermore, prevent hydrogen bonding to be established between particles and hence resist permanent deformation exerted under stress. This would result in a less mechanical interlocking and hence less mechanical strength of compressed tablets. Our investigation has suggested that at its best, in order to obtain powder compact with enough physical and mechanical integrity, not more than $12.5 \%$ of the lipidic oil can be included in the powder mix, i.e. $125 \mathrm{mg}$ of oil in $1 \mathrm{~g}$ weight tablet. There are two scenarios when it comes to the drug used for producing tablet SMEDDS; First: the drug needs to be administered in an amorphous dissolved state in the lipid matrix. In this case the, the maximum amount of oil which is allowed to be included in powder mix is $125 \mathrm{mg}$ which should be able to contain required dose. For example, if the therapeutic dose of a drug is $20 \mathrm{mg}$ and the solubility of the drug in an oil formulation is $100 \mathrm{mg} / \mathrm{g}$ lipid, then at least a $200 \mathrm{mg}$ of lipid needs to be added to the powder mix and hence, in this case, compaction will be compromised. Therefore, tablet SMEDDS technology can be applied for low therapeutic index drugs such as sirolimus or glyburide which are given at doses $<3 \mathrm{mg}$. In the second scenario, lipidic oil is added to the powder compact to influence release patterns, or inhibit P-gp efflux and CYP3A4 metabolism when a compound is a substrate for either efflux transporters or hepatic uptake.

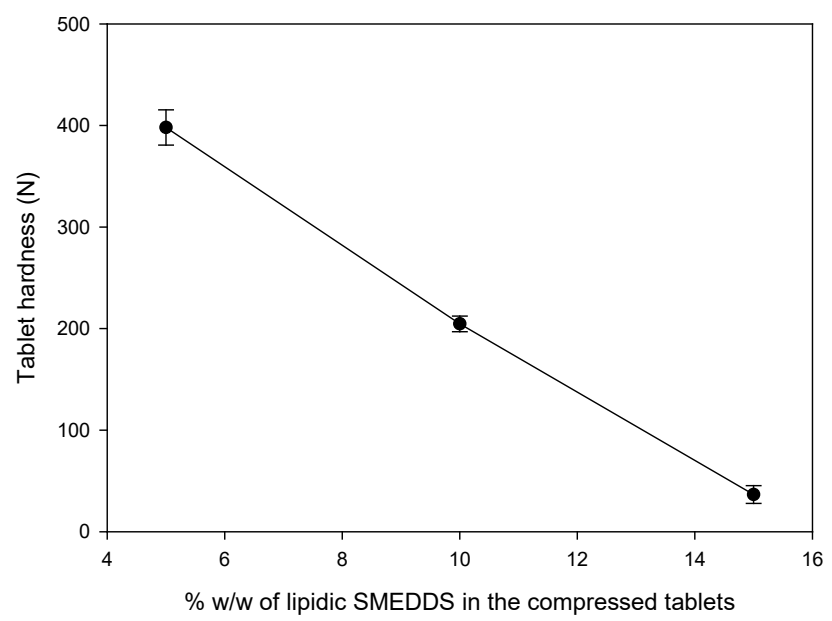

Fig. 5: Effect of adding increasing amounts of oil in the powder mix on the hardness profiles of compressed tablets.

\section{Release profiles of S-SMEDDS containing Theophylline as a BCS class I model compound}

Theophylline is a xanthine derivative used in the treatment of asthma. The drug is completely absorbed after the oral administration. Because of its short half-life ( 3 to $8 \mathrm{~h}$ ), theophylline is administered as extended-release dosage forms for better patient compliance. There are many different commercially available theophylline products on the market including; Uniphyllin (200 mg tablets), Nuelin SA (175 mg tablets) Euphyllin long (300 mg, capsules) and Slo-Phyllin (125 mg, capsules). Initially, there was no intention in this investigation to produce a sustained release system yet to study the effect of the lipidic SMEDSS added to the 
powder compact on the dissolution kinetics of theophylline tablets. The amount of theophylline which was added to the powder mix was approximately equivalent $125 \mathrm{mg}$. Figure 7 shows the disintegration patterns of various compressed SMEDDS tablets with and without drug at varying oil concentrations and different compaction forces. Compressed SMEDDS tablets of hardness values of $200 \mathrm{KN}$ (triangle up symbol) has shown relatively high disintegration times in comparison to tablet compacts with hardness values of $100 \mathrm{KN}$ (square or circle symbols). In comparison to compressed tablets containing no oil, generally, adding lipidic SMEDDS to the powder mix up to $5 \% \mathrm{w} / \mathrm{w}$ has resulted in decreases in designation times followed by increases at higher oil concentration for both systems with and without drug. Therefore, optimum disintegration times can be achieved at using oil concentration of 5\% w/w. Disintegration times for compressed tablets which have hardness values force of $100 \mathrm{KN}$ and using oil loading factor of $\{1: 9\}$ (i.e. oil concentration of $5 \%$ in the powder mix) without drug (circle symbol) or with drug (square symbol) were approximately; 1 or 3 minutes, respectively. Therefore, fast dissolving formulations were obtained by just including $5 \%$ lipidic oil in the powder mix. Furthermore, at using oil loading factor of $\{2: 8\}$ (i.e. oil concentration in the powder mix is $10 \%$ ), disintegration times for compressed tablets without drug or with were approximately; 3 or 21 minutes, respectively. Generally, the amount of drug and the oil content which is added to the powder compacts appear to influence dissolution and disintegration kinetics of SMEDDS tablets. Nonetheless, an optimum amount of $5 \%$ of lipidic SMEDDS is required to aid disintegration process which the contour plot depicted in Figure 8 confirms. As the amount of added oil increases in the powder mix, tablet disintegration time increases. The disintegration profiles presented in Figure 7 completely reflect dissolution patterns observed for theophylline from the various tested formulations which is shown in Figure 9. Theophylline dissolved at relatively higher rate from tablet compacts containing 5\% lipidic SMEDDS in comparison to powder compacts with 0 or $10 \% \mathrm{w} / \mathrm{w}$ oil. Therefore, adding $5 \%$ of SMEDDS oil to the powder compacts accelerates dissolution of theophylline and moreover ensues in producing fast dissolving tablet formulations. After 10 minutes, almost $80 \%$ of theophylline dissolved when $5 \%$ of SMEDDS was added to the powder mix in comparison to dissolved amounts of 60 or $50 \%$ of theophylline from compacts containing either 0 or $10 \% \mathrm{w} / \mathrm{w}$ lipidic SMEDDS, respectively. It appears that adding more oil to the powder $\operatorname{mix}(>5 \% \mathrm{w} / \mathrm{w})$ would retard dissolution of theophylline. It is anticipated that using 5\% w/w of lipidic SMEDDS which contains surfactant in the oil matrix will enhance wetting of powder and facilitate water uptake and thus improve disintegration and dissolution profiles of theophylline tablets. On the other hand, as the amount of SMEDDS oil builds up in the powder compacts, the prime preference of the lipidic SMEDDS is to adsorb water and utilize to emulsify first which ensues in delaying disintegration and hence release patterns of the drug.

Table 2: In vitro kinetic values of theophylline release from various types of formulations; see Table 1 for formulations composition. $\mathrm{K}_{0}\left[\mathrm{mg} \times \mathrm{m}^{-1}\right]:$ zero-order rate constant, $\mathrm{K}_{1}\left[\mathrm{~m}^{-1}\right]$ : first-order rate constant, $\mathrm{K}_{\mathrm{H}}\left[\mathrm{mg} \times \mathrm{m}^{-1 / 2}\right]$ : Higuchi rate constant, $\mathrm{K}_{\mathrm{HC}}\left[\mathrm{m}^{-1}\right]$ : Hixson Crowell rate constant, $\mathrm{K}_{\mathrm{KP}}\left[\mathrm{m}^{-\mathrm{n}}\right]$ : Korsmeyer-Peppas rate constant, $\mathrm{n}$ : diffusion exponent, $\mathrm{r}^{2}$ : regression correlation coefficient.

\begin{tabular}{|c|c|c|c|c|c|c|c|c|c|c|c|}
\hline \multirow{3}{*}{ 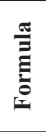 } & \multicolumn{11}{|c|}{ Kinetics model } \\
\hline & \multicolumn{2}{|c|}{ Zero-order } & \multicolumn{2}{|c|}{ First-order } & \multicolumn{2}{|c|}{ Higuchi } & \multicolumn{2}{|c|}{ Hixson-Crowell } & \multicolumn{3}{|c|}{ Peppas-Korsmeyer } \\
\hline & $\mathbf{r}^{2}$ & $k_{0}$ & $\mathbf{r}^{2}$ & $\mathbf{k}_{1}$ & $\mathbf{r}^{2}$ & $\mathbf{k}_{\mathrm{H}}$ & $\mathbf{r}^{2}$ & $\mathbf{k}_{\mathrm{HC}}$ & $\mathbf{r}^{2}$ & $\mathbf{k}_{\mathrm{PK}}$ & $n$ \\
\hline F0 & 0.732 & 2.03 & 0.958 & 0.059 & 0.939 & 15.21 & 0.903 & 0.062 & 0.806 & 2.45 & 1.161 \\
\hline F1 & 0.542 & 1.91 & 0.851 & 0.071 & 0.884 & 16.33 & 0.745 & 0.066 & 0.763 & 2.78 & 1.166 \\
\hline $\mathrm{F} 2$ & 0.807 & 1.16 & 0.964 & 0.047 & 0.982 & 14.5 & 0.923 & 0.053 & 0.839 & 2.32 & 1.156 \\
\hline
\end{tabular}

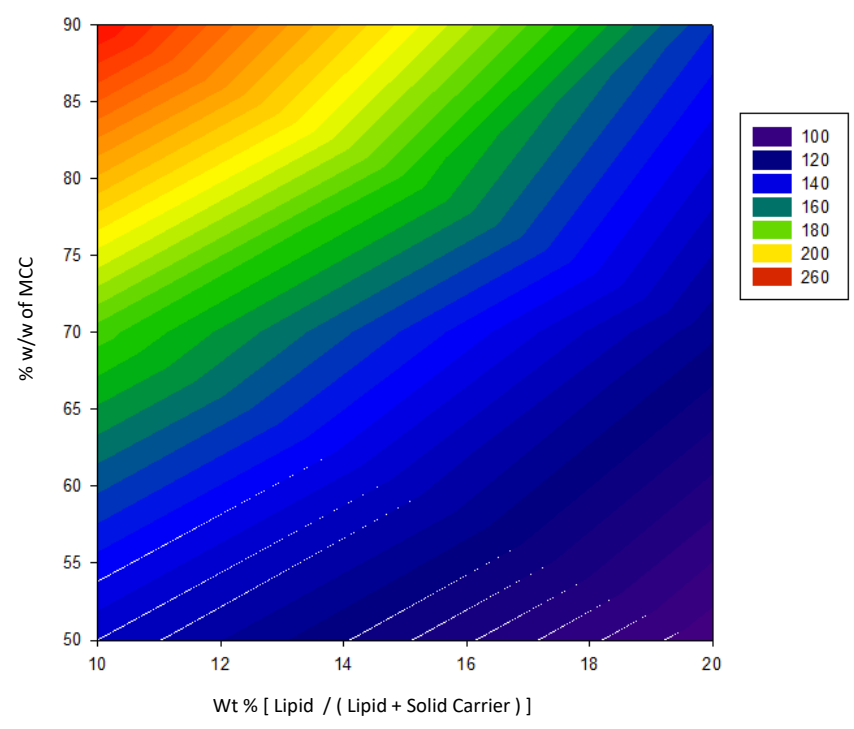

Fig. 6: Contour plot showing the effect of the progressive inclusion of increasing amounts of oil adsorbed unto the solid carrier on tablet hardness.
In order to study release kinetics of theophylline from various formulations, data obtained from In vitro drug release studies were plotted in various kinetic models: zero-order, first-order, Higuchi model, Hixson-Crowell, and KorsmeyerPeppas. As Tablet 2 suggests, all examined kinetics models predict relatively faster dissolution rates for tablets which contain $5 \% \mathrm{w} / \mathrm{w}$ lipidic oil in comparison to powder compacts containing either 0 or $10 \% \mathrm{w} / \mathrm{w}$ SMEDDS oil. Furthermore, analysis of the dissolution kinetic data for tested theophylline preparations shows that it follows first-order or Higuchi kinetics; see Table 2 and figure 10. Nonetheless, better regression correlation coefficients were observed in the case of systems containing 0 or $10 \% \mathrm{w} / \mathrm{w}$ lipidic oil which reflects that fast dissolving nature of SMEDDS tablets containing 5\% oil.

Due to the hydrophobic nature of MCC or magnesium trisilicate hydrate, we anticipate that the drug release occurs by dissolution and diffusion of the drug through water-filled capillaries within the matrix's pore network (Crowley et al., 2004). According to the relative rates of diffusion $\left(\mathrm{R}_{\text {diff }}\right)$ 
and polymer relaxation $\left(\mathrm{R}_{\text {relax }}\right)$ four classes of diffusion can be distinguished (Druzynska and Czubenko, 2012). The diffusional exponent, $n$, is dependent on the geometry of the delivery system and the physical mechanism for release. For a cylindrical geometry, the value of $i$ ) $n \leq 0.45$ indicates a Fickian diffusion mechanism (Case I); $\mathrm{R}_{\text {diff }}$ « $\mathrm{R}_{\text {relax }}$, system controlled by diffusion, ii) $0.5<n<1.0$ indicates non-Fickian (anomalous) diffusion mechanism; $\mathrm{R}_{\text {diff }} \approx \mathrm{R}_{\text {relax }}$, iii) $n=1.0$ indicates Case II, $\mathrm{R}_{\text {diff }}$ " Rrelax, system controlled by relaxation, $i v$ ) values of $n>1$ are regarded as Super Case II kinetics (Donglu, 2004). It can be clearly seen from Table 2 that values of the diffusional exponent $(n)$ for all tested formulations are $>1$ or close to 1.0 which indicates that the transport mechanism is Super Case II or Case II (relaxation controlled).

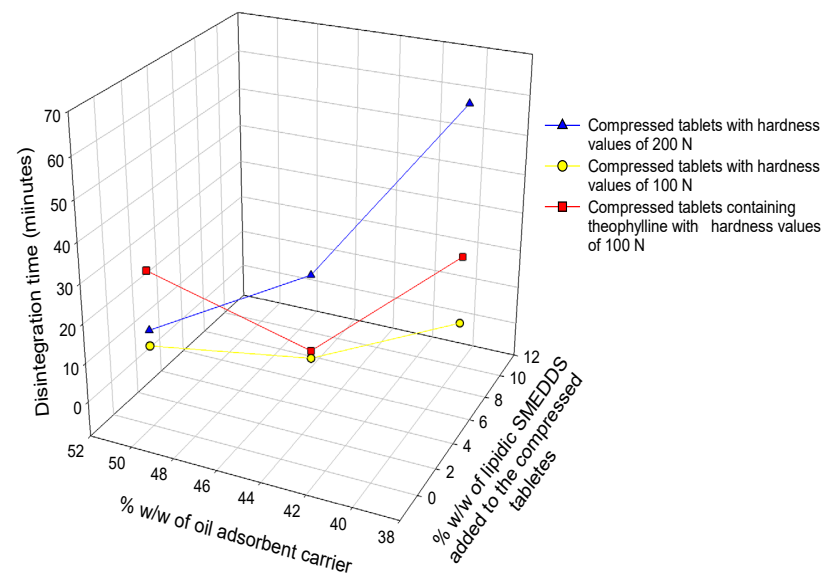

Fig. 7: Disintegration patterns of various compressed SMEDDS tablets with and without drug at varying oil concentrations and different loading compaction forces.

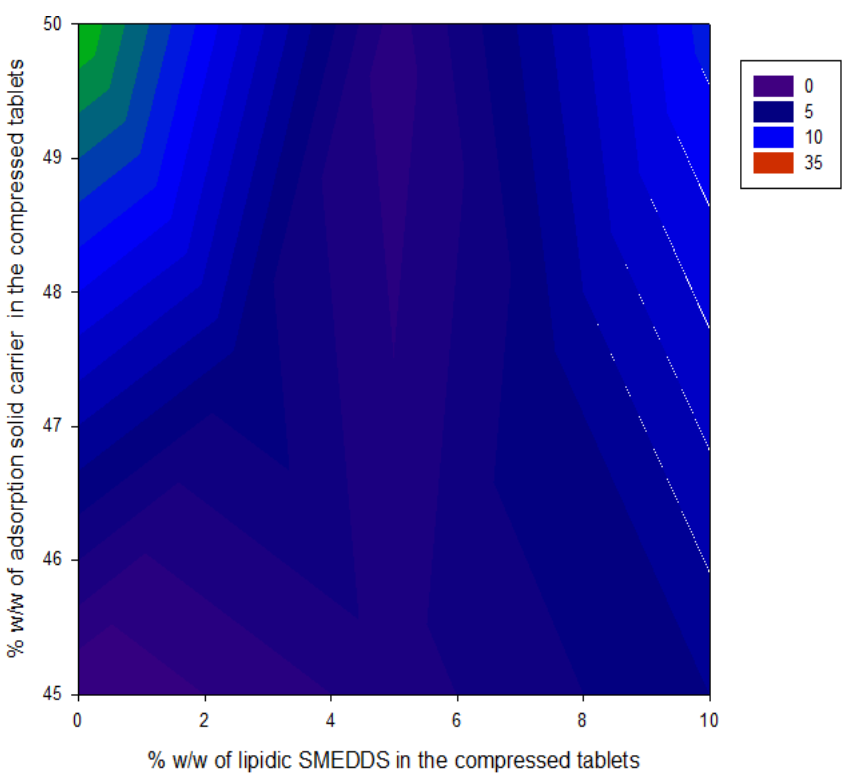

Fig. 8: Contour plot showing the effect of the progressive inclusion of increasing amounts of oil which is adsorbed onto the solid carrier on average disintegration time of tablets.

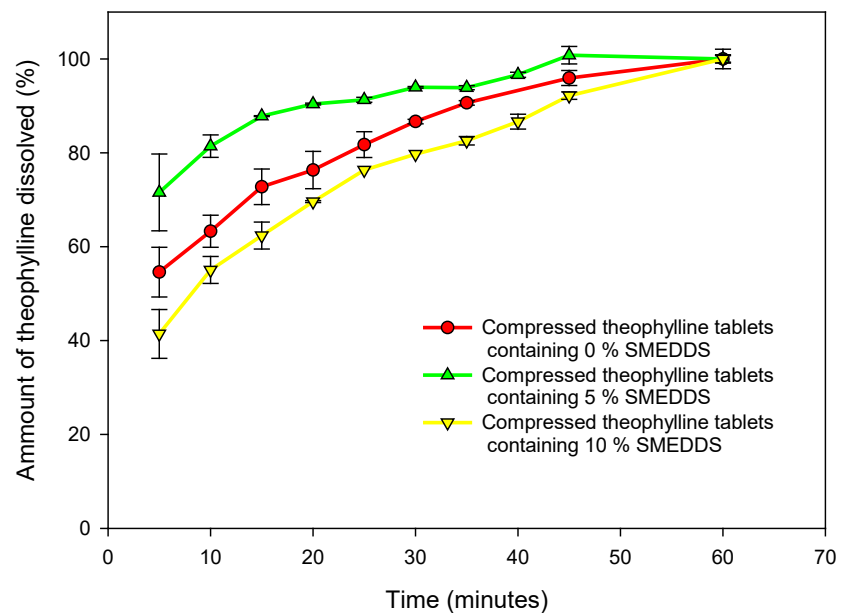

Fig. 9: Dissolution profile observed for theophylline from the various tested formulations.

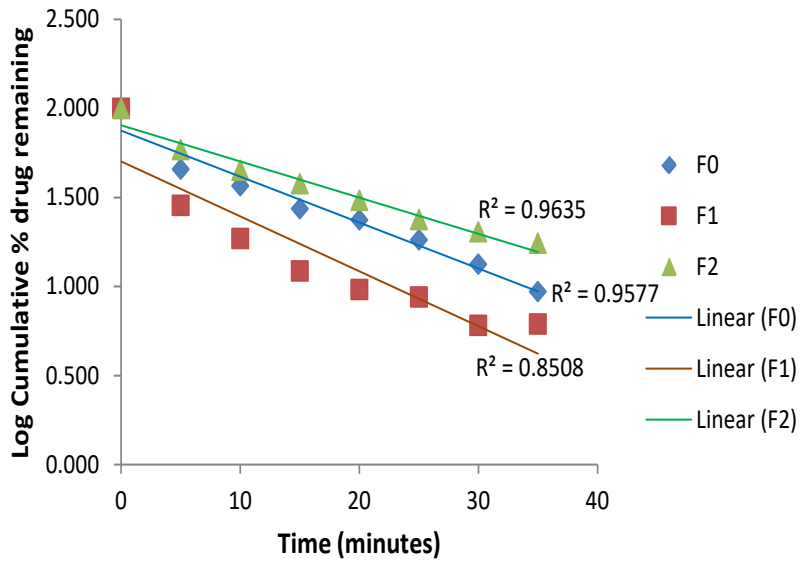

Fig. 10: Linear plots for the dissolution data from the various types of tested formulations in accordance with the first-order kinetics. (See table 1 for formulations composition).

\section{CONCLUSIONS}

A self-micro-emulsifying oil formulation representing type IIIA lipid was converted into solid SMEDDS using Magnesium trisilicate hydrate (MTSH) as solid adsorption carriers and MCC as a binder. The inclusion of increasing amounts of oil SMEDDS in the powder compacts affects mechanical strength and release patterns of theophylline tablets. Fast dissolving tablet formulations were obtained at including only optimum oil concentration of 5\% w/w. It is anticipated that using $5 \% \mathrm{w} / \mathrm{w}$ of lipidic SMEDDS can enhance wetting of powder and facilitate water uptake and thus improve disintegration and dissolution profiles of theophylline tablets. Furthermore, analysis of the dissolution kinetic data for tested theophylline preparations shows that it follows first-order or Higuchi kinetics.

\section{ACKNOWLEDGMENTS}

We are grateful to the Faculty of Pharmacy at Taif University for providing a good atmosphere and excellent facilities 
of research. We are also grateful to Croda for sending samples as gifts. Finally, my gratitude's go to the school of Pharmacy at Applied Science Private University for their support.

\section{REFERENCES}

Abdulla A, Klein S, Mäder K. A new self-emulsifying drug delivery system (SEDDS) for poorly soluble drugs: Characterization, dissolution, In vitro digestion and incorporation into solid pellets. Europ J Pharmac Sci, 2008; 35(5):457-64.

Bansal T, Akhtar N, Jaggi M, Khar RK, Talegaonkar S. Novel formulation approaches for optimizing the delivery of anti cancer drugs based on p-glycoprotein modulation. Drug Discov Today, 2009; 14:1067-74.

Brouwers J, Mols R, Annaert P, Augustijns P. Validation of a differential in situ perfusion method with mesenteric blood sampling in rats for intestinal drug interaction profiling. Biopharm Drug Dispos, 2010; 31(56):278-85.

Butler JM, Dressman JB. The developability classification system: Application of biopharmaceutics concepts to formulation development. J Pharm Sci, 2010; 99:4940-54.

Buyukozturk F, Benneyan JC. Carrier Impact of emulsion-based drug delivery systems on intestinal permeability and drug release kinetics. J Control Release, 2010; 142:22-30.

Chambin O, Jannin V, Champion D, Chevalier C, RochatGonthier MH, Pourcelot Y. Influence of cryogenic grinding on properties of a self-emulsifying formulation. Int J Pharm, 2004; 278(1):79-89.

Chatterjee B, Almurisi SH, Dukhan AAM, Mandal UK, Pinaki SP. Controversies with self-emulsifying drug delivery system from pharmacokinetic point of view. Drug Deliv, 2016; 23(9):3639-52.

Christiansen ML, Holm R, Kristensen J, Kreilgaard M, Jacobsen $\mathrm{J}$, Abrahamsson B, et al. Cinnarizine food-effects in beagle dogs can be avoided by administration in a self nanoemulsifying drug delivery system (SNEDDS). Eur J Pharm Sci, 2014; 57:164-72.

Crowley MM, Schroeder B, Fredersdorf A, Obara S, Talarico M, Kucera S, McGinity JW. Physicochemical properties and mechanism of drug release from ethyl cellulose matrix tablets prepared by direct compression and hot-melt extrusion. Int J Pharm, 2004; 269:509-522.

Donglu S. Biomedical Devices and Their Applications. Beijing, China, Springer, 2004, pp 1-31.

Druzynska MG, Czubenko JO. Mechanism of water diffusion into noncrosslinked and ionically crosslinked chitosan membranes. Prog Chem Appl Chit Deriv, 2012; 17:59-66.

Eng JG, Xu C. Development of solid self-emulsifying drug delivery systems: preparation techniques and dosage forms. Drug Discov Today, 2008; 13(13-14):606-12.

Farah N, De Teddeo M, Larfrêt JP, Denis J. 1993. Selfmicroemulsifying drug delivery system for improving in-vitro dissolution of drugs. AAPS Annual Meeting, Orlando, FL.

Gohel MC. Novel drug delivery approaches to bypass Pglycoprotein efflux pump. Pharmainfo net, 2011; http://www.pharmainfo. net/reviews/novel-drug-delivery-approaches-bypass-p-glycoprotein-effluxpump.

Gregory Thoorens G, Krier F, Leclercq B, Carlin B, Evrard B. Microcrystalline cellulose, a direct compression binder in a quality by design environment-A review. Int J Pharm, 2014; 473:64-72.

Hasan NMY. Preparation of Solid Self-Micro-Emulsified Lipid Systems for the Delivery of Hydrophobic Drugs. Int J Pharm Res, 2015a; $7(3): 75-84$.

Hasan MYN. 2004. Self-micro-emulsifying lipid formulations to improve the bioavailability of poorly water-soluble drugs. Ph.D. thesis, University of Bath.

Hasan NMY, Al-aram MSA, Al-wadie MSM, Althobaiti FAK, Al-Malki MJA. Flavored self microemulsifying lipid formulations for masking the organoleptic taste of pharmaceutical actives. J Appl Pharm Sci, 2015b; 5(11):127-34.

Hasan NMY, Almalki DM, Althuwaybi MJK, Alshehri
HM. SMEDDS tablet: compatability of solid smedds using various pharmaceutical tablet excipients. Int J Pharm Pharm Sci, 2016; 8(9):24651.

Hasan NMY, Hayajneh FM, Khaleel MA, Alharthi SA, Shahada HM, Almalki HF. Development of Potential Self-microemulsifying Lipid Formulation for the Oral Administration of Curcumin. Int J Adv Pharm Biol Chem, 2015c; 4(3):590-602.

Inugala S, Eedara BB, Sunkavalli S, Dhurke R, Kandadi P, Jukanti $\mathrm{R}$, et al. Solid self-nanoemulsifying drug delivery system (S-SNEDDS) of darunavir for improved dissolution and oral bioavailability: In vitro and in vivo evaluation. Eur J Pharm Sci, 2015; 74:1-10.

Jannin V, Musakhanian J, Marchaud D. Approaches for the development of solid and semi-solid lipid-based formulations. Adv Drug Deliv Rev, 2008; 60(6):734-46.

Kang MJ, Jung SY, Song WH, Park JS, Choi SU, Oh KT, et al. Immediate release of ibuprofen from Fujicalin-based fast-dissolving selfemulsifying tablets. Drug Dev Ind Pharm, 2011; 37:1298-305.

Khairnar DA, Darekar AB, Saudagar RB. A review on self-micro emulsifying drug delivery system: evident to improve the oral bioavailability of hydrophobic drug. Asian J Pharm Tech, 2016; 6(2):131-34.

Kim DW, Kang JH, Oh DH, et al. Development of novel flurbiprofen-loaded solid self-microemulsifying drug delivery system using gelatin as solid carrier. J Microencapsul, 2012; 29:323-30.

Kim DW, Kwon MS, Yousaf AM, Balakrishnan P, Park JH, Kim DS, et al. Comparison of a solid SMEDDS and solid dispersion for enhanced stability and bioavailability of clopidogrel napadisilate. Carbohydr Polym, 2014; 114:365-74.

Laddha P, Suthar V, Butani S. Development and optimization of self microemulsifying drug delivery of domperidone. Braz J Pharm Sci, 2014; 50(1):91-100.

Nankervis R, Davis SS, Day NH, Shaw PN. Effect of lipid vehicle on the intestinal lymphatic transport of isotretinoin in the rat. Inter $\mathrm{J}$ Pharm, 1995; 119(2):173-81.

Nornoo AO, Zheng H, Lopes LB, Johnson-Restrepo B, Kannan $\mathrm{K}$, Reed R. Oral microemulsions of paclitaxel: In situ and pharmacokinetic studies. Eur J Pharm Biopharm, 2009; 71:310-7.

Okimoto K, Miyake M, Ibuki R, Yasumura M, Ohnishi N, Nakai T. Dissolution mechanism and rate of solid dispersion particles of nilvadipine with hydroxypropylmethylcellulose. Int J Pharm, 1997; 159:8593

Porter CJ, Kaukonen AM, Boyd BJ, Edwards GA, Charman WN. Susceptibility to lipase-mediated digestion reduces the oral bioavailability of danazol after administration as a medium-chain lipid-based microemulsion formulation. Pharm Res, 2004; 21(8):1405-12.

Pouton CW. Formulation of poorly water-soluble drugs for oral administration: physicochemical and physiological issues and the lipid formulation classification system, Eur J Pharm Sci, 2006; 29:278-87.

Quan Q, Kim DW, Marasini N, Kim DH, Kim JK, Kim JO, et al. Physicochemical characterization and in vivo evaluation of solid selfnanoemulsifying drug delivery system for oral administration of docetaxel J Microencapsul, 2013; 30:307-14.

Ramasahayam B, Eedara BB, Kandadi P, Jukanti R, Bandari $\mathrm{S}$. Development of isradipine loaded self-nano emulsifying powders for improved oral delivery: In vitro and in vivo evaluation. Drug Dev Ind Pharm, 2015; 41:753-63.

Sander C, Holm P. Porous Magnesium Aluminometasilicate Tablets as Carrier of a Cyclosporine Self-Emulsifying Formulation. AAPS Pharm Sci Tech, 2009; 10(4):1388-95.

Schwartz JB, Lachman L. 1990. Compressed Tablets by direct compression. In: Bandelin JF, editor. Pharmaceutical Dosage Forms: Tablets Vol. 1. New York, Basel, HongKong: Marcel Dekker Inc; p. 158.

Setthacheewakul S, Mahattanadul S, Phadoongsombut N, Pichayakorn W, Wiwattanapatapee R. Development and evaluation of self-microemulsifying liquid and pellet formulations of curcumin, and absorption studies in rats. Europ J Pharm Biopharm, 2010; 76(3):475-85.

Shah NH, Carvajal MT, Patel, CI, Infeld MH, Malick AW. Self- 
emulsifying drug delivery system (SEDDS) with polyglycolyzed glycerides for improving In vitro dissolution and oral absorption of lipophilic drugs. Int J Pharm, 1994; 106:15-23.

Shanmugam S, Baskaran R, Balakrishnan P, Thapa P, Yong CS, Yoo BK. Solid self-nanoemulsifying drug delivery system (S-SNEDDS) containing phosphatidylcholine for enhanced bioavailability of highly lipophilic bioactive carotenoid lutein. Eur J Pharm Biopharm, 2011; 79:250-7.

Sharma S, Khinchi MP, Sharma N, Agrawal D, Gupta MK. Approaches to development of solid-self micron emulsifying drug delivery system: formulation techniques and dosage forms - A review. Asian J Pharmac Res Dev, 2013; 1(5):146-56.

Sha X, Wu J, Chen Y, Fang X. Self-microemulsifying drugdelivery system for improved oral bioavailability of probucol: preparation and evaluation. Inter J Nanom, 2012; 7:705-12.

Shraddha DP, Nayan AG, Bhushan RR, Sunil PP. Self-micro emulsifying drug delivery system (smedds): a promising drug delivery system for enhancement of bioavailability. Ind J Drugs, 2016; 4(3):90-108.

Tang G, Ch Liang LL, Yi T, Lam CWK. Effects of SprayDrying and Choice of Solid Carriers on Concentrations of Labrasol ${ }^{\circledR}$ and Transcutol ${ }^{\circledR}$ in Solid Self-Microemulsifying Drug Delivery Systems (SMEDDS). Molecules, 2013; 18:545-60.
Zhao X, Zhou YQ, Potharaju S, Lou H, H. Sun M, Brunson E, et al. Development of a self micro-emulsifying tablet of cyclosporine- A by the liquisolid compact technique. IJPSR, 2011; 2(9):2299-308.

U.S. Department of Health and Human Services Food and Drug Administration, waiver of in vivo bioavailability and bioequivalence studies for immediate-release solid oral dosage forms based on a biopharmaceutics classification system guidance for industry; Center for Drug Evaluation and Research (CDER), Biopharmaceutics. 2015.

US Food and Drug Administration. Guidance for Industry, waiver of in vivo Bioavailability and Bioequivalence Studies for Immediate-Release Solid Oral Dosage Forms Based on a Biopharmaceutics Classification System; Center for Drug Evaluation and Research: Rockville, MD, USA, 2000.

How to cite this article:

Hasan NMY, Khaleel MA, Altwairqi AS, Alqurashi AG, Altwairqi AH. Effect of Self-Microemulsifying Lipid Formulations on the Dissolution and Compaction Profiles of Tablets Containing Theophylline; a BCS Class I Compound. J App Pharm Sci, 2018; 8(06): 030-038. 